\section{CPS-251 EVALUATION OF CLOSTRIDIUM DIFFICILE INFECTION MANAGEMENT PRIOR TO IMPLEMENTATION OF A PROTOCOL FOR IMPROVEMENT}

${ }^{1} V$ Acín Garcés, ${ }^{2} \mathrm{MM}$ Vinuesa Hernando, ${ }^{2} \mathrm{R}$ Gracia Piquer, ${ }^{2} \mathrm{R}$ Fresquet Molina, ${ }^{3} \mathrm{E}$ Morte Romea, ${ }^{2} \mathrm{M}$ Gimeno*, ${ }^{4} \mathrm{C}$ Seral García, ${ }^{2} \mathrm{M}$ Arenere Mendoza. ${ }^{1}$ Universidad De Zaragoza, Medicine Faculty, Zaragoza, Spain; ${ }^{2}$ Hospital Clínico Universitario Lozano Blesa, Pharmacy, Zaragoza, Spain; ${ }^{3}$ Hospital Clínico Universitario Lozano Blesa, Infectious Diseases, Zaragoza, Spain; ${ }^{4}$ Hospital Clínico Universitario Lozano Blesa, Microbiology, Zaragoza, Spain

\subsection{6/ejhpharm-2021-eahpconf.83}

Background and importance Clostridium difficile disease (CDD) is the leading cause of infectious diarrhoea in the hospital environment.

Aim and objectives The objective was to evaluate the characteristics of patients with CDD prior to the implementation of a checklist for the diagnosis and treatment of CDD.

Material and methods This was a retrospective observational study of CDD cases in a third level hospital during 2019. Positive cases were evaluated according to the checklist: vulnerability (cancer, neutropenic, transplant, inflammatory bowel disease (IBD) or prolonged antibiotic treatment), severity (according to leucocytosis, renal function or presence of hypotension, shock or ileus), risk of recurrence (according to age, CDD the previous year, positive toxin or persistence of diarrhoea on the fifth day of treatment), treatment and antibiotic intake in the previous 3 months.

Results 126 cases of CDD were identified in 100 patients, with a median age of 76 (1-96), and 59\% were women. $19.8 \%$ of the cases were vulnerable (5 transplants, 3 IBD, 8 oncological and 1 immunosuppressed). Severity of the episodes was assessed in 116 patients, with 62.9\% not serious, 31.9\% serious and $5.2 \%$ (6) fulminant. The fulminant cases were hypotension/shock (4) and megacolon (2). The risk of recurrence was high in $10.8 \%$, intermediate in $55.0 \%$ and low in $34.2 \%$. Of the 100 patients diagnosed, 21\% (21) presented with at least one recurrence and 4\% (4) with multiple recurrences. In addition, $7 \%$ had some episodes in the previous years. Regarding treatment of the 126 episodes of CDD: $20.6 \%$ were treated with metronidazole, $58.7 \%$ with vancomycin $(8.7 \%$ followed by vancomycin taper), $7.2 \%$ with metronidazole + vancomycin, $3.9 \%$ with fidaxomycin, $3.2 \%$ with metronidazole + vancomycin + fidaxomycin, $0.8 \%$ with metronidazole+fidaxomycin, $0.8 \%$ with vancomycin+fidaxomycin and $4.8 \%$ were not treated as they were considered to be self-limiting processes. In $82.5 \%$, the patient received at least one antibiotic in the previous 3 months.

Conclusion and relevance Patients with CDD were older (76 years). Despite the low vulnerability of the population, severe and fulminant cases reached almost $40 \%$. Treatment was heterogeneous, but metronidazole and vancomycin remained the standard of care. In $82 \%$ of cases, patients had received prior antibiotic treatment, an aspect of particular relevance to the future management of CDD, as $21 \%$ of patients presented with recurrence.

\section{REFERENCES AND/OR ACKNOWLEDGEMENTS}

Conflict of interest No conflict of interest

\section{CPS-252 STUDY OF THE IMPLEMENTATION OF A VANCOMYCIN PHARMACOKINETIC MONITORING PROGRAMME IN PAEDIATRICS}

${ }^{1} \mathrm{M}$ Pérez*, ${ }^{1} \mathrm{M}$ Merchante, ${ }^{1} \mathrm{~L}$ Cazorla, ${ }^{1} \mathrm{~A}$ Magallón, ${ }^{1} \mathrm{~A}$ Pinilla, ${ }^{1} \mathrm{~J}$ Perales, ${ }^{1} \mathrm{~A}$ López,

2J Bueno. 'Universitary Hospital Miguel Servet, Hospital Pharmacy, Zaragoza, Spain; ${ }^{2}$ Universitary Hospital Miguel Servet, Psychiatry, Zaragoza, Spain

\subsection{6/ejhpharm-2021-eahpconf.84}

Background and importance Efficacy and toxicity are strictly related to serum concentrations of vancomycin. Special care must be considered when treating paediatric patients with this antibiotic. Monitoring and adjusting to protocols and guidelines are key tools for a successful therapy.

Aim and objectives To analyse compliance with the vancomycin dosage protocol in paediatric patients in a third level hospital and to evaluate the impact of pharmacokinetic monitoring on the adequacy of therapeutic levels.

Material and methods A descriptive, observational, retrospective study was conducted from the beginning of the implementation of the vancomycin monitoring programme (January 2017) until August 2020. It included all paediatric patients who had received recommendations for dosage adjustment after determination of vancomycin serum levels. Demographic and clinical variables were collected. Adherence to the initial dosage of the protocol, serum levels at the beginning of the monitoring programme and serum levels at the end of treatment were studied.

Results Data were collected from a total of 133 paediatric patients, 83 males (62.4\%). Mean age was calculated for two subpopulations, neonatal patients $(<1$ month old $)$ with a mean age of $14.5 \pm 9.4$ days and paediatric patients $(>1$ month old) with a mean age of $4.4 \pm 4.2$ years (1 month-14 years). $68.1 \%$ of the neonatal patient population were premature, at less than 40 weeks' gestation. The main services that requested monitoring were the neonatal ICU (40.6\%), paediatric ICU $(27.8 \%)$, neonates $(14.3 \%)$, paediatrics infectious diseases $(8.3 \%)$, paediatrics $(5.3 \%)$ and paediatric oncology $(3.8 \%)$. Reported treatment diagnoses were: suspected infection $(37.2 \%)$, sepsis $(25.6 \%)$, meningitis $(10.9 \%)$, bacteraemia (7.8\%), pneumonia (4.7\%), gastrointestinal infection $(3.9 \%)$, urinary tract infection (1.6\%) and other causes $(8.5 \%)$.

Adherence to the protocol prior to monitoring was $81.2 \%$. Vancomycin levels were within the therapeutic range in $30.8 \%$ of cases at the start of monitoring. Following pharmacokinetic recommendations, the values were within the range in $66.2 \%$ of cases.

Conclusion and relevance The variability in the paediatric patients in this study showed that, even though most prescriptions were in accordance with the protocol, only $30.8 \%$ achieved therapeutic levels. This percentage doubled after the monitoring programme, which highlights the great value of monitoring and personalised dose recommendations, especially recommended in this type of patient.

\section{REFERENCES AND/OR ACKNOWLEDGEMENTS}

Conflict of interest No conflict of interest 